\title{
Transient Waterhouse-Friderichsen Syndrome in a Child: A Case Report
}

\author{
Gian Luca Trobia ${ }^{\mathrm{a}}$, Federica Sullo ${ }^{\mathrm{b}, \mathrm{e}}$, Maria Carla Finocchiaro ${ }^{\mathrm{a}}$, Eloisa Gitto $^{\mathrm{c}}$, Carmen Damiano ${ }^{\mathrm{c}}$, \\ Rita Maria Caccamo ${ }^{\mathrm{d}}$, Rosario Ranno ${ }^{\mathrm{d}}$, Vita Antonella Di Stefano ${ }^{\mathrm{a}}$
}

\begin{abstract}
Waterhouse-Friderichsen syndrome (WFS) is a rare but usually fatal disease characterized by adrenal insufficiency associated to bilateral adrenal hemorrhage. It can be associated both to non-infection and a variety of infectious diseases, among which sepsis from Neisseria meningitidis accounts for more than $80 \%$ of cases. We report the case of an otherwise healthy 2-year-old child who had a WFS as a consequence of Neisseria meningitidis infection and in which we witnessed a complete recovery of adrenal function a few months after the event. The case highlights not only the difficulty of diagnosing this syndrome but also, at the same time, the extreme importance of high suspicion, early treatment, a multidisciplinary approach and, most of all, the importance of verifying the restoration of adrenal function in order to achieve a better quality of life.
\end{abstract}

Keywords: Waterhouse-Friderichsen syndrome; Neisseria meningitidis; Adrenal insufficiency; Pediatric emergency room; Purpuric lesions; Fever

\section{Introduction}

Waterhouse-Friderichsen syndrome (WFS), first described in 1911 by Rupert Waterhouse and Carl Friderichsen, is an uncommon, but usually fatal disease characterized by adrenal insufficiency associated to bilateral adrenal hemorrhage. This

Manuscript submitted January 24, 2021, accepted February 12, 2021

Published online March 19, 2021

aUnit of Pediatrics and Pediatric Emergency Care, Cannizzaro Emergency Hospital, Catania, Italy

bDepartment of Clinical and Experimental Medicine, Section of Pediatrics and Child Neuropsychiatry, University of Catania, Catania, Italy

'Neonatal Intensive Care Unit, Department of Pediatrics, University of Messina, Policlinico G. Martino, Messina, Italy

dUnit of Intensive Burn Centre, Cannizzaro Emergency Hospital, Catania, Italy

${ }^{\mathrm{e} C}$ Corresponding Author: Federica Sullo, Department of Clinical and Experimental Medicine, Section of Pediatrics and Child Neuropsychiatry, University of Catania, Catania, Italy. Email: fedesullo@hotmail.it

doi: https://doi.org/10.14740/ijcp429 syndrome can be associated both to non-infection and a variety of infectious diseases, among which sepsis from Neisseria meningitidis accounts for more than $80 \%$ of WFS cases. It is more common in children compared to adults and, although the pathophysiology is not fully understood, various theories have been proposed. The case we are going to describe is about a 2-year-old child, who experienced a WFS as a consequence of Neisseria meningitidis infection and in which we witnessed a complete recovery of adrenal function, thanks to high suspicion and early diagnosis.

\section{Case Report}

A previously healthy 2-year-old child was brought to our pediatric emergency room for sudden appearance of purpuric lesions in the lower limbs associated with fever and pharyngitis. The patient had not performed all the vaccinations recommended by the national calendar and he did not have any significant health problems up to date. Pregnancy and delivery were normal. He had normal psychomotor milestones, except for a slight language delay. After evaluation in our emergency department (ED), his vital signs showed a prolonged refill time (4 s), blood pressure $110 / 55 \mathrm{~mm} \mathrm{Hg}$, and elevated heart rate to 145 beats $/ \mathrm{min}$. He was febrile at $39^{\circ} \mathrm{C}$, and his respiratory rate was $32 \mathrm{breaths} / \mathrm{min}$. As regards neurological examinations, he was awake and oriented but appeared irritable, weak and fatigued. The cranial nerves appeared unscathed, the osteotendinous reflexes were normally elicitable and the pupils were isochoric, isocyclic and normoreactive to the light stimulus. Walking was normal for age. His skin was pale, with petechiae and suffusions bleeding spread on the face, trunk and limbs. His examination was otherwise normal, with the exception of hyperemia of the pharynx. Laboratory investigations, including complete blood count, biochemical parameters, arterial bloodgas and coagulation profile, were then performed. The results were the following (values in parentheses are the reference ranges): $\mathrm{Hb} 11.7 \mathrm{~g} / \mathrm{dL}$, leukocyte $4,200 / \mathrm{mm}^{3}$, neutrophil $75.2 \%$, platelet $194,000 / \mathrm{mm}^{3}$, C-reactive protein 11.6 $\mathrm{mg} / \mathrm{dL}(<1 \mathrm{mg} / \mathrm{dL})$, procalcitonin $67.7 \mathrm{ng} / \mathrm{mL}(>10 \mathrm{ng} / \mathrm{mL}$, high risk of septic shock), urea $27 \mathrm{mg} / \mathrm{dL}(10-55 \mathrm{mg} / \mathrm{dL})$, creatinine $0.55 \mathrm{mg} / \mathrm{dL}(0.40-1.30 \mathrm{mg} / \mathrm{dL})$, glucose $125 \mathrm{mg} /$ $\mathrm{dL}(65-120 \mathrm{mg} / \mathrm{dL})$, sodium $137 \mathrm{mEq} / \mathrm{L}(134-150 \mathrm{mEq} / \mathrm{L})$ and potassium $3.3 \mathrm{mEq} / \mathrm{L}(3.6-5.5 \mathrm{mEq} / \mathrm{L})$. Arterial blood gas analysis showed $\mathrm{pH} 7.41, \mathrm{pCO}_{2} 27 \mathrm{~mm} \mathrm{Hg}, \mathrm{pO}_{2} 82 \mathrm{~mm}$ 


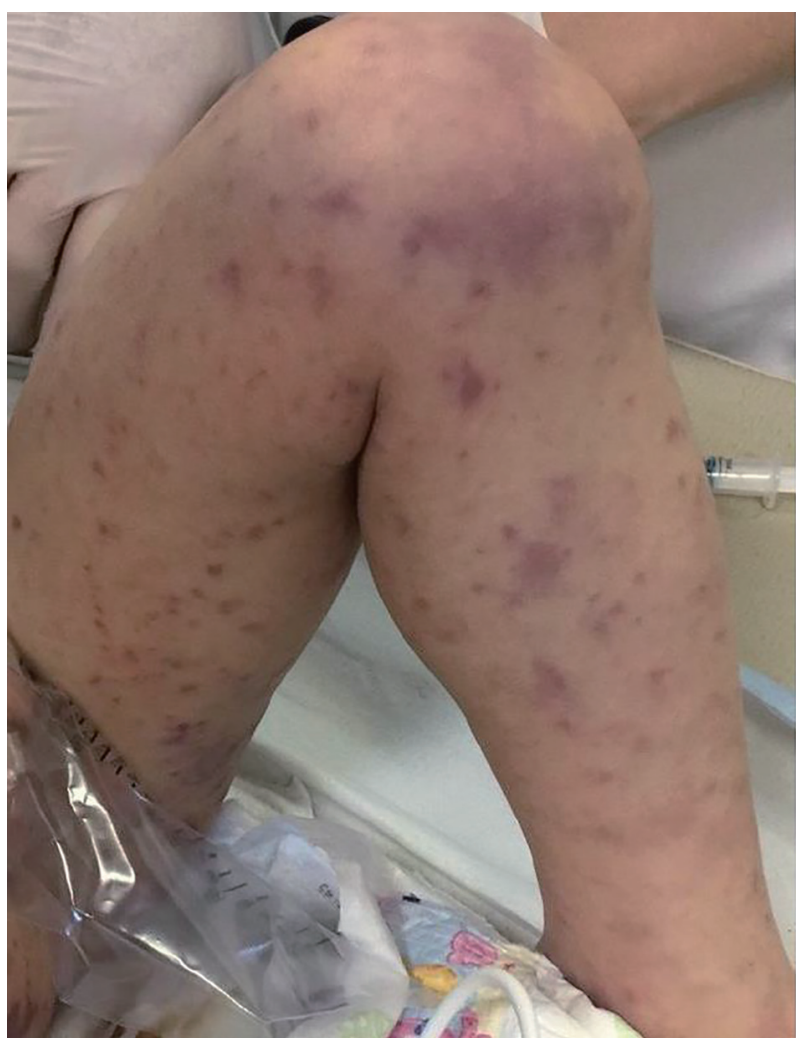

Figure 1. Extremely rapid numerical increase in skin lesions in the emergency room.

$\mathrm{Hg}$, lactic acid 7.1, $\mathrm{HCO}_{3}-19.5$ and base excess (BE) -7.25. A blood culture was also performed which was then negative. Waiting for results, and in the suspicion of meningococcal infection, we decided to undertake steroid and antibiotic therapy with ceftriaxone $(100 \mathrm{mg} / \mathrm{kg} /$ day $)$. During his residence in the emergency room, the child presented with an extremely rapid numerical increase in skin lesions (Fig. 1), despite absence of meningeal signs and normal vital parameters. He was then transferred to the pediatric intensive care where, after sedation, he was intubated and subjected to continuous intravenous therapy with fentanyl $(2 \mu \mathrm{g} / \mathrm{kg} / \mathrm{h})$ and midazolam $(2 \mu \mathrm{g} / \mathrm{kg} / \mathrm{min})$ for 10 days. In the suspicion of meningococcal sepsis, infusion of negative O Rhesus (RH) plasma, antithrombin III, vitamin $\mathrm{K}$ and triple intravenous therapy with ceftriaxone $(100 \mathrm{mg} / \mathrm{kg} /$ day), acyclovir $(45 \mathrm{mg} / \mathrm{kg} / \mathrm{day})$ and dexamethasone $(0.6 \mathrm{mg} /$ $\mathrm{kg}$ /day) were used for 3 days. At the same time, hematochemical sampling was performed to evaluate inflammation indices, hepato-renal function and coagulation screening. After antimicrobial therapy was started, he also underwent a lumbar puncture with culture test and Gram's stains, which showed a clear cerebrospinal fluid with normal biochemical findings (glucose $151 \mathrm{mg} / \mathrm{dL}$, proteins $2.1 \mathrm{~g} / \mathrm{dL}$, cell count $3 / \mathrm{mm}^{3}$ ), but it was discovered the presence of Neisseria meningitidis, with real time polymerase chain reaction (PCR) method. The child then stopped antiviral therapy, after 3 days, and continued targeted intravenous therapy with meropenem $(112 \mathrm{mg} / \mathrm{kg} /$ day $)$ and ceftriaxone $(100 \mathrm{mg} / \mathrm{kg} /$ day $)$ for 10 days. After admission, two blood cultures were taken, both negative. Due to the onset

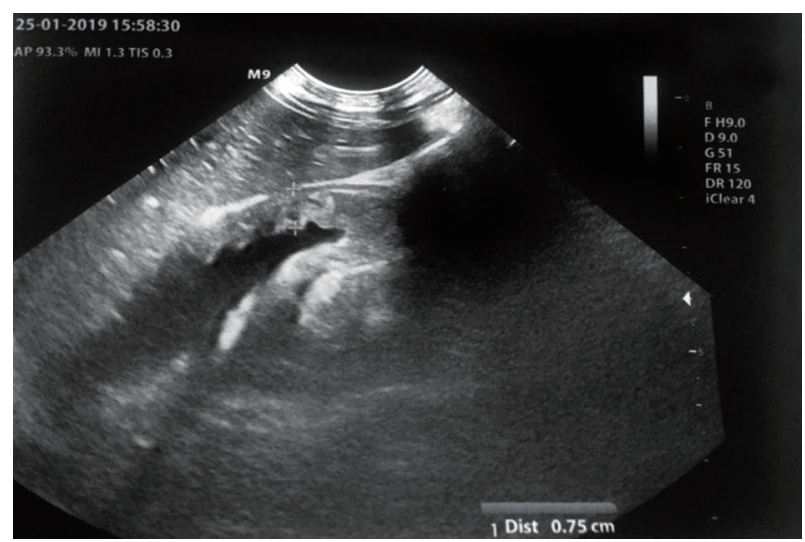

Figure 2. Ultrasonography of adrenal glands.

of disseminated intravascular coagulation (DIC) and diagnosis by laboratory investigations (platelet $81,000 / \mathrm{mm}^{3}$, fibrinogen $232 \mathrm{mg} / \mathrm{dL}$, prothrombin (PT) 30\%, international normalized ratio (INR) 2.22, prothrombin time (PTT) $97.1 \mathrm{~s}$, D-dimer > $4 \mu \mathrm{g} / \mathrm{mL}$ ), it became necessary to practice therapy with protein $\mathrm{C}$ concentrate $(4,000 \mathrm{U} /$ day for 4 days, then 2,000 U/day for 2 days) associated to plasma infusions. Inotropics such as dopamine $(5 \mu \mathrm{g} / \mathrm{kg} / \mathrm{min})$ and noradrenaline $(0.3 \mu \mathrm{g} / \mathrm{kg} / \mathrm{min})$ have also been administered for 5 days to support the circulation, now worsened. The clinical picture was complicated by the appearance of bilateral adrenal hemorrhage, diagnosed by ultrasonography (Fig. 2), and adrenal insufficiency, such as WFS. Adrenal insufficiency was confirmed when his adrenocorticotrophin (ACTH) measurement resulted at $1,202 \mathrm{pg} / \mathrm{mL}$ $(4.7-48.8 \mathrm{pg} / \mathrm{mL})$ and cortisol level $<18 \mu \mathrm{g} / \mathrm{dL}$. It was therefore necessary to start replacement therapy with hydrocortisone and fludrocortisone, initially intravenously at high doses $\left(100 \mathrm{mg} / \mathrm{m}^{2}\right.$ body surface area) and subsequently orally at the minimum effective dose. On subsequent days, he was able to maintain normal electrolyte levels and he was discharged after a 2-month hospitalization, on the following treatment plan: hydrocortisone $12 \mathrm{mg} / \mathrm{m}^{2} /$ day and fludrocortisone acetate 0.1 $\mathrm{mg}$ /day. The patient's first follow-up visit was 1 month after discharge and subsequently every 2 weeks with hormonal and electrolyte monitoring (Table 1). After 6 months, a complete restoration of the adrenal function was obtained which allowed reduction and finally suspension of the therapy. As a result of meningococcal sepsis, our patient presented a deterioration of skin lesions (Fig. 3), and unfortunately, vast areas of skin necrosis in upper and lower limbs and some smaller ones in the face (Fig. 4). During hospitalization, several escharectomy surgical sessions and subsequent coverage with auto-grafts were performed by the intensive burn center. After escharectomies, necrotic lesions involving the joint structures of the left elbow and the left knee were protected with dermal substitutes. At the last follow-up, he presented hypertrophic skin scars (Fig. 5). Finally, for the detection of psychomotor retardation, our patient performed a neuropsychiatric evaluation which highlighted a compromise in the areas of language and communication and hand-eye coordination. For this reason, he actually follows a speech and psychomotor therapy with benefit. A brain magnetic resonance imaging (MRI) study was performed and it did 
Table 1. Laboratory Follow-Up

\begin{tabular}{|c|c|c|c|c|c|}
\hline Follow-up date & $\begin{array}{l}\text { ACTH, pg/mL } \\
(4.7-48.8)\end{array}$ & $\begin{array}{l}\text { Cortisol, } \mu \mathrm{g} / \mathrm{dL} \\
(6.70-22.60)\end{array}$ & $\begin{array}{l}\text { Glucose, mg/ } \\
\text { dL (65 - 120) }\end{array}$ & $\begin{array}{l}\text { Sodium, mEq/L } \\
(134-150)\end{array}$ & $\begin{array}{l}\text { Potassium, } \mathrm{mEq} / \mathrm{L} \\
(3.6-5.5)\end{array}$ \\
\hline 2 months & 4.1 & 0.01 & 86 & 139 & 5.5 \\
\hline 3 months & 9.9 & 0.30 & 76 & 136 & 4.6 \\
\hline 4 months & 30.8 & 1.60 & 78 & 135 & 4.5 \\
\hline 5 months & 36.8 & 4.40 & 84 & 129 & 4.6 \\
\hline 8 months & 10 & 12.30 & 80 & 140 & 5.2 \\
\hline
\end{tabular}

ACTH: adrenocorticotrophin.

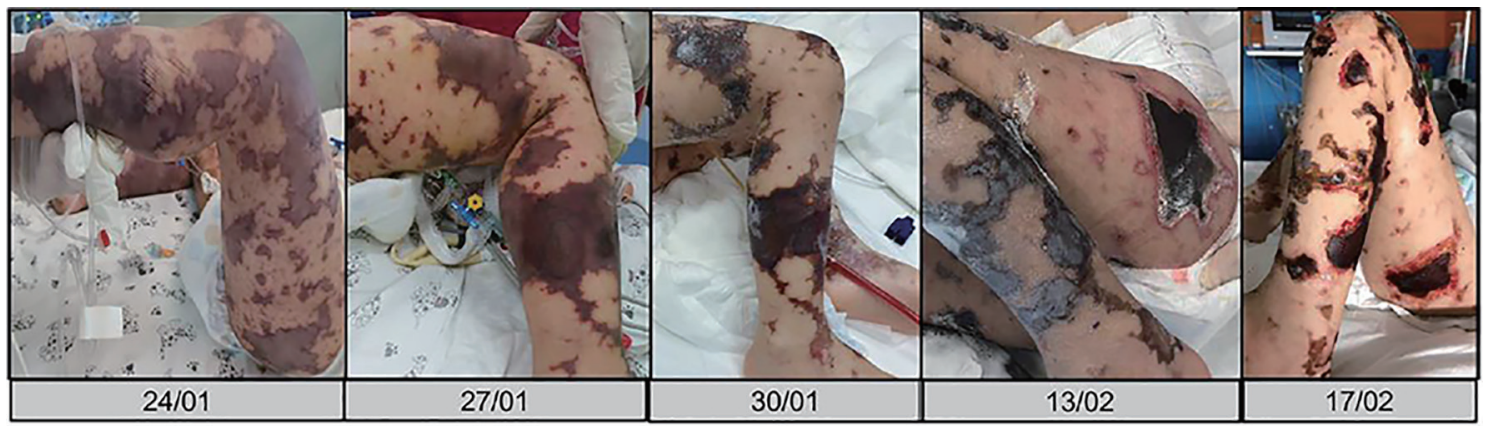

Figure 3. Skin lesions evolution in the pediatric intensive care unit.

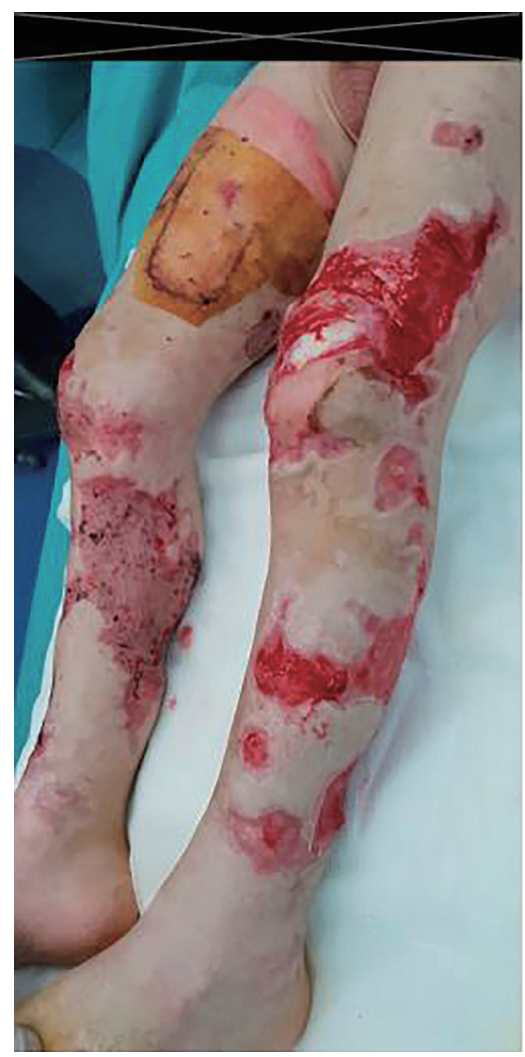

Figure 4. Skin ulcer in lower limb.

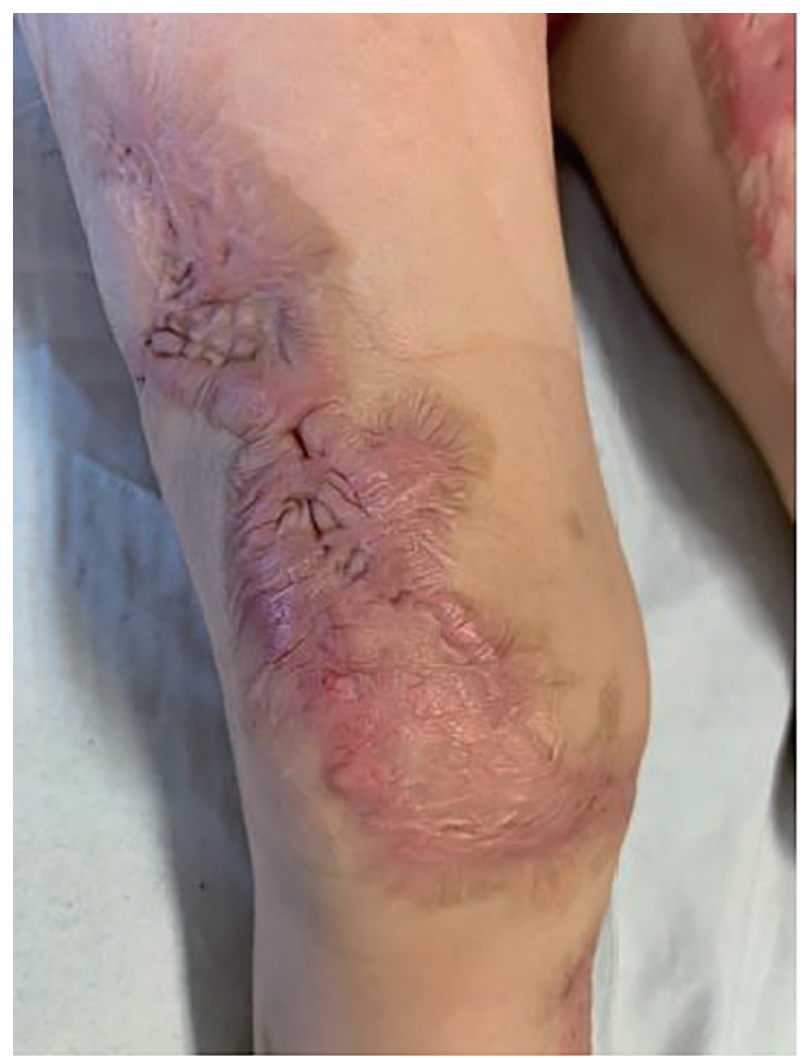

Figure 5. Hypertrophic skin scars after escharectomies and skin grafts. 
not show any lesions compatible with meningitidis outcomes.

\section{Discussion}

In this case report, we described the case of a 2-year-old patient who came to the emergency room for fever, pharyngitis and sudden onset of purpura in the lower limbs. The child presented a rapid increase in the number of lesions in the absence of signs of shock, which forced him to be transferred to a pediatric intensive care unit where, following a lumbar puncture, he was diagnosed with sepsis due to Neisseria meningitidis. The clinical picture was further complicated by the onset of DIC and bilateral adrenal bleeding, such as WFS. The literature reports cases of this syndrome more frequently in adulthood and often with a clinical onset characterized by a picture of shock as well as an often poor prognosis. Our case, in addition to being pediatric, underlines the importance of an early diagnosis and a high clinical suspicion that allowed the patient to survive albeit with notable scarring and mild psychomotor retardation.

WFS is a rare, but usually fatal consequence of Neisseria Meningitidis sepsis, characterized by adrenal insufficiency associated to bilateral adrenal hemorrhage [1]. Despite this predominant association, over the years it has been demonstrated that WFS may occur in association to other well recognized etiologies, such as sepsis due to different organisms, as well as to non-infectious causes, such as thromboembolic diseases, anticoagulant treatment [2], antiphospholipid syndrome [3], burns, trauma, tumor metastasis, cardiovascular catastrophes and postoperative state. Regarding infectious causes, several bacteria have been correlated, both Gram-positive and Gramnegative, such as Escherichia coli [4], Hemophilus influenzae, Streptococcus pneumonia [5], Staphylococcus aureus [6], group A Streptococcus [7], Neisseria gonorrhoeae [8], Pseudomonas aeruginosa [9], Enterobacter cloacae [10]. WFS has also been reported with Rickettsia rickettsii, Treponema pallidum, Legionella pneumophila and many different viral infections such as cytomegalovirus, Epstein-Barr virus, varicella zoster virus and parvovirus [11]. In 2019, Ventura Spagnolo et al described the case of a healthy 40-year-old man who died of severe multiorgan failure with WFS due to Proteus mirabilis infection [12]. WFS is more common in children than in adults and, although the pathophysiology is unclear, different theories have been postulated. Endotoxin-mediated septic shock is the most frequent clinical condition seen in patients with infectious adrenal hemorrhage. These endotoxins activate a large number of endothelial and inflammatory cells, inducing a number of signaling pathways that lead to transcription of coagulation, fibrinolysis, and pro-inflammatory cytokine genes activation (as tissue factor, interleukin (IL)-1, IL-6, and tumor necrosis factor-alpha) [13]. But another mechanism is involved which is the increased synthesis of cortisol, including adrenaline, by the adrenal gland in any stressful condition.

From an anatomical point of view, it must be remembered that adrenal gland is a vulnerable organ in which any cause leading to an increase in the adrenal venous pressure would lead to intraglandular hemorrhage. The increased serum adrenaline induces platelet aggregation in the adrenal veins and this adds up to the increased pressure within the vessels induced by ACTH which raises adrenal blood flow [14]. Adrenal insufficiency is associated to considerable morbidity and mortality, frequently connected to lack of awareness or education regarding its management. The clinical presentation can be, in fact, gradual and nonspecific to hypotension or shock, so-called adrenal crisis, depending on the degree of insufficiency and precipitating stress events. Symptoms include fever, weakness, fatigue, anorexia, nausea or vomiting, myalgias and arthralgias [15]. The principal manifestation of WFS is shock and, when it is with meningococcemia, it characteristically demonstrated petechial rash which usually develops on the trunk and lower portions of the body, but it can also involve the mucous membranes. If no treatment is administered, patients may develop vomiting, seizure, disorientation, mental status changes, circulatory collapse and shock [16]. Blood chemistry investigation may document leukocytosis, and because of underlying bacterial infection, an elevated anion gap metabolic acidosis. Coagulation abnormalities are consistent with DIC and consumption of clotting factors [17]. The lack of cortisol negative feedback increases hypothalamic corticotropin-releasing hormone $(\mathrm{CRH})$, leading to increased pituitary ACTH. Aldosterone deficiency causes sodium loss, which leads to electrolyte abnormalities including hyponatremia, hyperkalemia and metabolic acidosis. Hypoglycemia may be a presenting symptom and it can be associated with ketosis, due to impaired gluconeogenesis and hepatic glycogenesis. A mild increase of azotemia may also be present, as a consequence of volume contraction. It is also necessary to have Gram's stains and cultures from urine, blood and cerebrospinal fluid. Computed tomography (CT) scan of the abdomen is considered as the "gold standard" in the diagnosis of adrenal hemorrhage [18].

To assess adrenal function levels of ACTH, cortisol, aldosterone and renin activity, as well as ACTH test results should be obtained. Due to the severity of clinical picture, treatment should be started immediately before obtaining results. Acute adrenal insufficiency must be treated urgently with sufficient parenteral hydrocortisone, $100-150 \mathrm{mg}$ or $100 \mathrm{mg} / \mathrm{m}^{2}$ intravenously, saline with dextrose to restore intravascular volume, normalized serum sodium and blood glucose concentration. Management also includes antibiotic coverages (penicillin $\mathrm{G}$ or third-generation cephalosporin), vasopressor and other supportive care. The difficulty in diagnosing the WFS is that it is easily confused with septic shock and hypovolemic shock. You have to suspect WFS if the shock does not respond to intravenous fluids and vasopressors. In any case the diagnosis requires a high degree of suspicion, because the prognosis depends on the timeliness of diagnosis and of the consequent treatment. Unfortunately, in cases of delay, the case fatality is almost 50\%, despite aggressive treatment.

In our case, the diagnosis of adrenal insufficiency was made on the basis of the ultrasound examination, associated with the pathognomonic laboratory findings, in the absence of abdominal CT which still represents the gold standard. Thanks to an early diagnosis and appropriate management, our patient recovered. In literature no long-term data assessing the continuing requirements for replacement therapy are available, so close follow-up and re-evaluation are necessary. In 2004, after follow-up of four patients with acute bilateral adrenal 
hemorrhage and glucocorticoid insufficiency from $6(1 / 2)$ to 19 years, Jahangir-Hekmat et al documented the absence of need for long-term mineralocorticoid replacement and some improvement in endogenous glucocorticoid function in at least some of their patients [19]. In our case, it was possible to reduce the replacement therapy until complete suspension on the basis of no occurrence of postural hypotension, normal electrolytes and hormones.

Unfortunately, it was not possible to determine the serum aldosterone levels as well as plasma renin activity levels. This represents the main limitation of our study but we believe this case may be useful to disseminate the message that WFS requires high suspicion, early treatment and a multidisciplinary approach.

\section{Conclusions}

To the best of our knowledge, this is the first case in literature associated to meningococcal sepsis in a child, followed by complete restoration of adrenal function a few months after the event. We think it could be useful and interesting to describe this case and also to underline the undisputed value of vaccination practice in the prevention of the main infectious diseases, and finally to confirm the importance of early diagnosis and timely therapeutic intervention in order to avoid the most severe evolutions, such as death and mutilation of the limbs. In conclusion, it also seems useful and important to reiterate the importance of studying adrenal function remotely in these subjects in order to detect a complete restoration and therefore to allow the patient a better quality of life.

\section{Acknowledgments}

None to declare.

\section{Financial Disclosure}

Authors have no financial relationships relevant to this article to disclose. No funding was secured for this study.

\section{Conflict of Interest}

Authors have no conflict of interest to disclose.

\section{Informed Consent}

Patient's informed consent for publication of this report was obtained.

\section{Author Contributions}

Dr. Trobia and Dr. Sullo conceptualized and designed the study, drafted the initial manuscript, and reviewed and revised the manuscript for important intellectual content. Dr. Finocchiaro and Dr. Di Stefano designed the data collection instruments, coordinated and supervised data collection, and critically reviewed the manuscript. Dr. Gitto, Dr. Caccamo and Dr. Ranno collected data, carried out the initial analyses, and reviewed and revised the manuscript. All authors approved the final manuscript as submitted and agreed to be accountable for all aspects of the work.

\section{Data Availability}

The authors declare that data supporting the findings of this study are available within the article.

\section{Abbreviations}

WFS: Waterhouse-Friderichsen syndrome; DIC: disseminated intravascular coagulation; IL: interleukin; ACTH: adrenocorticotrophin; RH: Rhesus; CT: computed tomography

\section{References}

1. Knight B. Sudden unexpected death from adrenal haemorrhage. Forensic Sci Int. 1980;16(3):227-229.

2. Rosenberger LH, Smith PW, Sawyer RG, Hanks JB, Adams RB, Hedrick TL. Bilateral ad-renal hemorrhage: the unrecognized cause of hemodynamic collapse associated with heparin-induced thrombocytopenia. Crit Care Med. 2011;39(4):833-838.

3. Espinosa G, Santos E, Cervera R, Piette JC, de la Red G, Gil V, Font J, et al. Adrenal in-volvement in the antiphospholipid syndrome: clinical and immunologic characteristics of 86 pa-tients. Medicine (Baltimore). 2003;82(2):106-118.

4. Khwaja J. Bilateral adrenal hemorrhage in the background of Escherichia coli sepsis: a case report. J Med Case Rep. 2017;11(1):72.

5. Fox B. Disseminated intravascular coagulation and the Waterhouse-Friderichsen syndrome. Arch Dis Child. 1971;46(249):680-685.

6. Adem PV, Montgomery CP, Husain AN, Koogler TK, Arangelovich V, Humilier M, Boyle-Vavra S, et al. Staphylococcus aureus sepsis and the WaterhouseFriderichsen syndrome in chil-dren. N Engl J Med. 2005;353(12):1245-1251.

7. Hamilton D, Harris MD, Foweraker J, Gresham GA. Waterhouse-Friderichsen syndrome as a result of non-meningococcal infection. J Clin Pathol. 2004;57(2):208-209.

8. Swierczewski JA, Mason EJ, Cabrera PB, Liber M. Fulminating meningitis with Water-house-Friderichsen syndrome due to Neisseria gonorrhoeae. Am J Clin Pathol. 1970;54(2):202-204.

9. Margaretten W, Nakai H, Landing BH. Septicemic adrenal hemorrhage. Am J Dis Child. 1963;105:346-351. 
10. Pode-Shakked B, Sadeh-Vered T, Kidron D, Kuint J, Strauss T, Leibovitch L. Waterhouse Friderichsen syndrome complicating fulminant Enterobacter cloacae sepsis in a preterm infant: the unresolved issue of corticosteroids. Fetal Pediatr Pathol. 2014;33(2):104-108.

11. Karki BR, Sedhai YR, Bokhari SRA. Waterhouse-Friderichsen syndrome. [Updated 2019 Dec 3]. In: StatPearls [Internet]. Treasure Island (FL): StatPearls Publishing; 2020 Jan. Available from: https:/www.ncbi.nlm.nih.gov/ books/NBK551510/.

12. Ventura Spagnolo E, Mondello C, Roccuzzo S, Stassi C, Cardia L, Grieco A, Raffino C. A unique fatal case of Waterhouse-Friderichsen syndrome caused by Proteus mirabilis in an immu-nocompetent subject: Case report and literature analysis. Medicine (Baltimore). 2019;98(34):e16664.

13. Levi M, Keller TT, van Gorp E, ten Cate H. Infection and inflammation and the coagula-tion system. Cardiovasc Res. 2003;60(1):26-39.

14. Piccioli A, Chini G, Mannelli M, Serio M. Bilateral massive adrenal hemorrhage due to sepsis: report of two cas- es. J Endocrinol Invest. 1994;17(10):821-824.

15. Bowden SA, Henry R. Pediatric Adrenal Insufficiency: Diagnosis, Management, and New Therapies. Int J Pediatr. 2018;2018:1739831.

16. Varon J, Chen K, Sternbach GL. Rupert Waterhouse and Carl Friderichsen: adrenal apo-plexy. J Emerg Med. 1998;16(4):643-647.

17. Kunzer W, Schindera F, Schenck W, Schumacher H. [Waterhouse-Friderichsen syndrome: differential diagnosis, pathogenesis and treatment with streptokinase]. Dtsch Med Wochenschr. 1972;97(8):270-273.

18. Munoz Corsini L, Delgado Arnaiz C, Garcia del Valle S, Reboto Cortes P, Lopez del Cas-tillo A. Postoperative bilateral adrenal hemorrhage: correlation between clinical and radiological signs. J Clin Anesth. 2008;20(8):605608.

19. Jahangir-Hekmat M, Taylor HC, Levin H, Wilbur M, Llerena LA. Adrenal insufficiency at-tributable to adrenal hemorrhage: long-term follow-up with reference to glucocorticoid and miner-alocorticoid function and replacement. Endocr Pract. 2004;10(1):55-61. 